\title{
Characterization of a bonding method for titanium-glass sealing
}

\author{
Sebastian Winkler ${ }^{1} \cdot$ Jan Edelmann $^{1}{ }^{1} \cdot$ Andreas Schubert $^{1,2}$
}

Received: 15 May 2020 / Accepted: 9 November 2020 / Published online: 20 November 2020

(c) The Author(s) $2020 \quad$ OPEN

\begin{abstract}
Applications for smart medical implants require hermetic and mechanically strong seals between functional and biocompatible materials. Hermetic seals between titanium Ti6AI4V and silica-based glass can be produced using a novel bonding method based on glass pressing at temperatures around the softening point. This paper presents investigation results for the tensile bond strength and the gas leak rate depending on the manufacturing process parameters. Notably, when using blasted surfaces, the tensile bond strengths reached $12 \mathrm{MPa}$ and good adherence with very low leakage due to the removed oxide layer and surface structure. The interface is analyzed and characterized by applying SEM methods related to the different adhesion mechanisms.
\end{abstract}

Keywords Glass · Glass-to-metal seals $\cdot$ Titanium $\cdot$ Hermetic seal

\section{Introduction}

Glass-to-metal seals are required in numerous technological fields such as aerospace engineering [1], battery technology [2], and medical engineering [3]. Mechanical strength and sealing quality are the significant properties that determine the performance of glass-to-metal bondings. The glass material and the metal alloy must be biocompatible for applications in medical engineering.

Implantable electronic devices such as pacemakers are encapsulated in order to protect the human body from potentially harmful substances. Titanium alloys combine mechanical strength with biocompatibility, making them the material of choice for most medical implants containing electronic systems. Figure 1 illustrates an implantable electronic system with a titanium housing. This implant contains an optical window for optical data communication with a control system located outside the body, as described in [4]. Other potential applications for optical windows are optical diagnosis systems. Silica-based glasses match the requirements for mechanical strength, biocompatibility, and optical transmission. Moreover, the hermetic sealing between the titanium housing and the glass window is a crucial part of the encapsulation system (Fig. 1).

Bonding of glass and metal is an old technology that can even be traced back to ancient Egypt, where decorative glass coatings were manufactured on metal surfaces. The technology applied for these coatings is based on glass melting and wetting of the metal surface, which was later referred to as enameling. Most industrial applications such as light bulbs or enameled cookware are produced by this common type of manufacturing process using high temperatures to melt the glass, allowing it to wet the metal surface [5]. Bonding of glass and titanium for medical and other applications is challenging and has been studied extensively [6-8].

Compared to the conventional glass melting approach, other bonding methods with low process temperatures have been introduced [9]. In the anodic bonding process [10], the glass remains in its solid state, and static electric fields create the glass-to-metal interface. This technology is used for microfluidic applications [11].

$\triangle$ Jan Edelmann, jan.edelmann@iwu.fraunhofer.de $\mid{ }^{1}$ Fraunhofer Institute for Machine Tools and Forming Technology, Fraunhofer IWU, Chemnitz, Germany. ${ }^{2}$ Professorship Micromanufacturing Technology, TU Chemnitz, Chemnitz, Germany. 




Fig. 1 Implantable medical implant with functional glass windows

This paper investigates an alternative glass-to-metal bonding technology, first introduced in [12], with process temperatures between conventional melting technologies and low-temperature bonding technologies. The interface created at medium temperatures allows glass to be formed by compressive forces. The investigations' objective comprises determining the influence of the relevant process parameters on the quality of the glass-to-metal seal.

\section{Setup and procedure to test bonding properties}

\subsection{Sample design}

The investigation of the alternative bonding method uses a combination of a titanium alloy and a silica-based glass. Titanium grade 5 (Ti6Al4V) is the most common material used for medical implants and combines high mechanical strength with biocompatibility. The glass type B270 by Schott is an optical crown glass and was selected as its thermal expansion coefficient $\left(10.3 \times 10^{-6} \mathrm{~K}^{-1}\right)$ is similar to that of Ti6Al4V $\left(9.5 \times 10^{-6} \mathrm{~K}^{-1}\right)$. The major glass components comprise $\mathrm{SiO}_{2}(69 \%), \mathrm{B}_{2} \mathrm{O}_{3}(11 \%), \mathrm{Na}_{2} \mathrm{O}(10 \%), \mathrm{K}_{2} \mathrm{O}$ $(6 \%)$, and $\mathrm{BaO}(3 \%)$.
Two different sample geometries were designed in order to fulfill the requirements for measuring both the mechanical strength of the glass-to-metal interface and its hermetic sealing quality (leak tightness). Figure 2a shows the cross-section of the axially symmetric bonding device used for the determination of the bond's tensile strength and consists of two titanium punches enveloping a cylindrical glass element of $3 \mathrm{~mm}$ thickness and a diameter of $15 \mathrm{~mm}$. This design allows the direct measurement of the tensile strength (Fig. $2 \mathrm{~b}$ ) without requiring any glue. The second sample design in Fig. 3 a consists of a titanium ring surrounding a glass cylinder of the same geometry, allowing the leak rate measurement through the glass-to-metal peripheral interface.

All titanium samples were produced by precision milling. The blasted surfaces in contact with the glass were roughened by blasting with glass powder (type 09-0010, Baltrusch \& Mütsch) in a microblasting tool. A tactile roughness instrument was applied for measuring the surface roughness, which resulted in $\mathrm{Ra}=0.3 \mu \mathrm{m}$ for the milled surface, and $\mathrm{Ra}=1.7 \mu \mathrm{m}$ for the blasted one.

\subsection{Bonding process: glass pressing}

The glass samples are placed in a pressing die with a $\mathrm{Ti}$ sample on both the bottom and the upper punch in a vacuum chamber mounted in a universal testing machine. The samples are heated to the process temperature $T_{\mathrm{p}}$ in an argon atmosphere at a pressure of 5 mbar. Compared to a vacuum, the low-pressure atmosphere of inert gas allows for better heat conductivity from the heated die into the glass sample. In addition, the partial pressure of oxygen is further reduced, which prevents the growth of an oxide layer.

Applying compressive forces on the die provides bonding of the Ti and glass samples. The two parts of the die
Fig. 2 Experimental setups and sample design for a $\mathrm{Ti}-$ glass bonding process (pressing) and $\mathbf{b}$ testing of the bond tensile strength (drawings not to scale) (a)

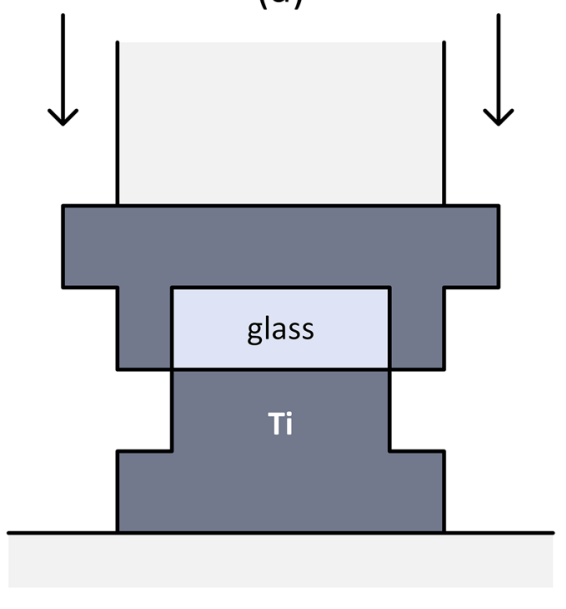

(b)

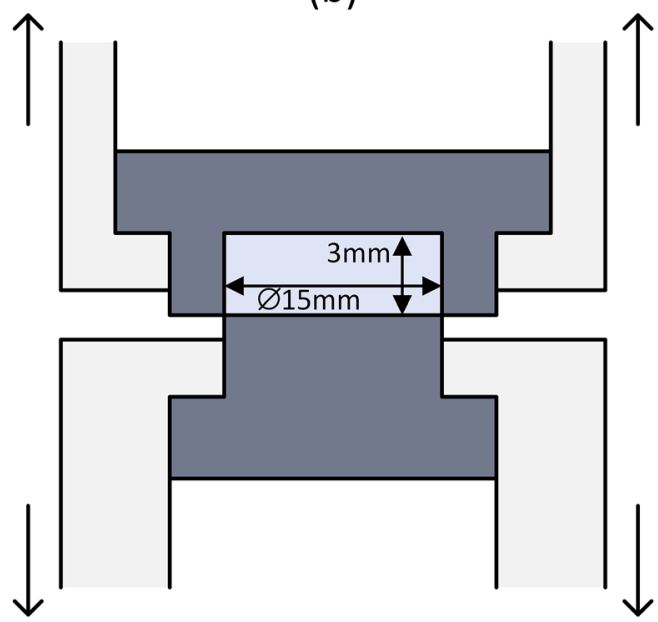


Fig. 3 Experimental setups, sample design for measurement of leak rate: glass pressing process (a), helium leak test (b), pressure gain test (c) (drawings not to scale) (a)

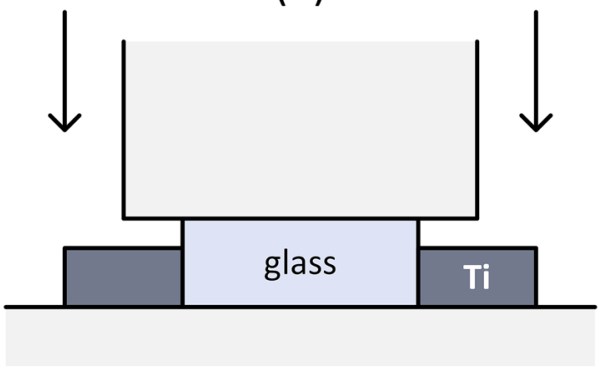

(c)

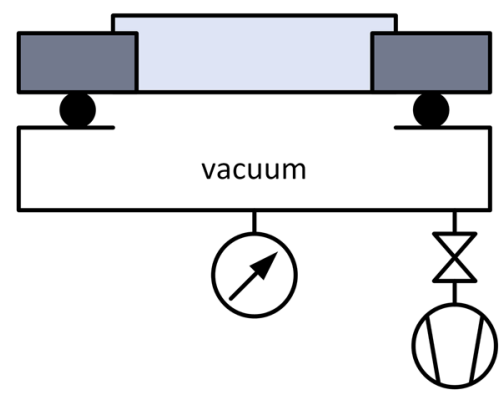

(b)

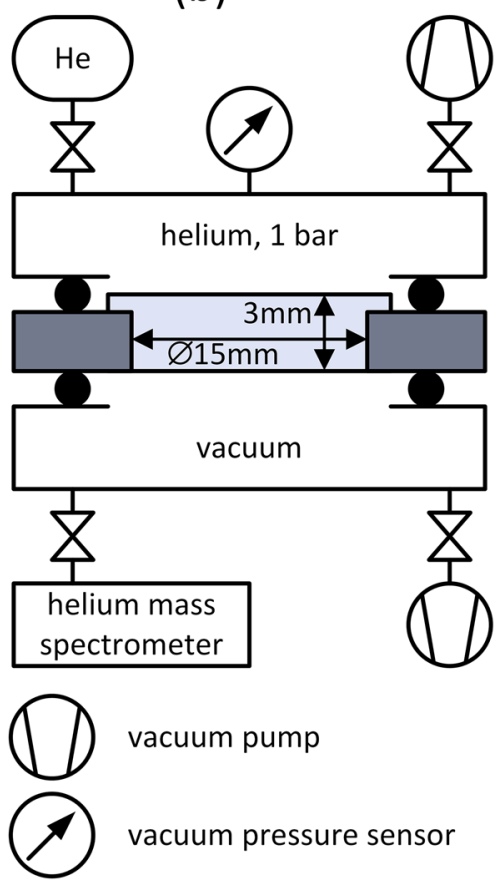

are equipped with a heater allowing for homogeneous temperature distribution. In the first sample geometry, the die touches the upper titanium sample, and the glass is pressed between the two titanium samples (Fig. 2a). In the second sample geometry, the upper punch directly touches the glass, which bonds to the ring-shaped titanium sample (Fig. 3a). These components are coated with a boron nitride layer to prevent the glass from bonding with the upper punch or the sample holder.

During pressing, the maximum process force $F_{p}$ is maintained for $15 \mathrm{~s}$. The applied bonding procedure and process parameters are identical for the two sample geometries. Therefore, the measurement results regarding tensile strength and gas leak rates are comparable to samples produced with the same process parameters. Winkler et al. [13] describe the layout of the glass-pressing tool and the individual process steps in detail.

The tested process temperatures of $T_{\mathrm{P}}=705^{\circ} \mathrm{C}, 745^{\circ} \mathrm{C}$, and $785^{\circ} \mathrm{C}$ cover a range of 1.5 orders of magnitude in viscosity around the softening point of the glass material B270 at $724^{\circ} \mathrm{C}$. The selected process forces $F_{\mathrm{p}}=200 \mathrm{~N}$, $500 \mathrm{~N}$, and $1200 \mathrm{~N}$ correspond to compressive stress values between 1 and $7 \mathrm{MPa}$, which is typical in glass pressing.

\subsection{Measurement of tensile strength and leak rate}

The adherence of a glass coating on a metal surface can be measured indirectly, e.g. by the Vickers indentation method [14], or directly with a pull-off test [15], in which the sample has to be glued to the testing machine. The tested device and the mechanical interlocking of the two titanium elements (Fig. 2b) are connected to the universal testing machine to avoid the difficulties of detecting the failure point associated with using glue. The tensile force increases in a linear rate of $20 \mathrm{~N} / \mathrm{s}$ up to $1000 \mathrm{~N}$ and $200 \mathrm{~N} / \mathrm{s}$ above this threshold. The maximum force before the breakage is registered, and the bond tensile strength $R_{\mathrm{H}}$ is calculated using the sample area of $177 \mathrm{~mm}^{2}(\varnothing 15 \mathrm{~mm})$.

The leak rate $Q_{L}$ is a measured parameter for hermetic sealing quality and is usually specified by the unit mbar. $\mathrm{l} / \mathrm{s}$. The size of the bonded area amounts to $141 \mathrm{~mm}^{2}$ in the experimental setup. At first, all samples are measured with a pressure gain test (Fig. $3 c$ ) as specified in DIN EN 1779. The vacuum chamber on the bottom side of the sample is evacuated, and then the vacuum pump is disconnected. The leak rate $Q_{L}$ can be calculated based on recording the rising pressure over time due to leakage through the glass-to-metal interface. The helium leak test is applied as a second measurement method [16] for samples with leak rates lower than $10^{-4} \mathrm{mbar} \mathrm{l} / \mathrm{s}$. Figure $3 \mathrm{~b}$ illustrates the experimental setup. The Ti sample's upper side is filled with helium under atmospheric pressure of $1 \mathrm{bar}$, while the bottom side is evacuated and connected to a mass spectrometer designed for the helium leak test. The test procedure is capable of measuring leak rates down to $10^{-12} \mathrm{mbar} \mathrm{l} / \mathrm{s}$. 


\section{Results and discussion}

\subsection{Tensile bond strength and leak rate}

Figures 4 and 5 present the results of the tensile strength $R_{H}$ and the leak rate $Q_{L}$ for the indicated parameter combinations. For most of the experiments, the tensile strength ranges from $7 \mathrm{MPa}$ to $12 \mathrm{MPa}$. Only three parameter combinations resulted in very low values for $R_{\mathrm{H}}$, which implies no adhesion. The leak rate results (Fig. 5) are similar to those of the tensile strength and are divided into two groups. The first group comprises leak rates of $1 \cdot 10^{-6} \mathrm{mbar} \cdot 1 / \mathrm{s}$ and below, which is considered technically hermetic, while the second group of leak rates amounts to more than $0.01 \mathrm{mbar} \cdot \mathrm{l} / \mathrm{s}$, which is considered non-hermetic.

In addition to measuring their tensile strength and leak rates, the samples were analyzed by scanning electron microscopy (SEM). Four of the leak-rate-samples with selected parameter combinations were cut perpendicular to the glass titanium interface, ground, polished, and cleaned before being analyzed by SEM. Figure 6 shows the SEM images together with the corresponding process parameters.

\subsection{Bonding mechanism}

Bonding can be defined as adhesion, which describes the connection between two dissimilar materials at their contact surface. Even today, numerous aspects of adhesion are still subject to research. Various adhesion models with mechanical, electrostatic, chemical, or thermodynamic approaches are available for describing the effects

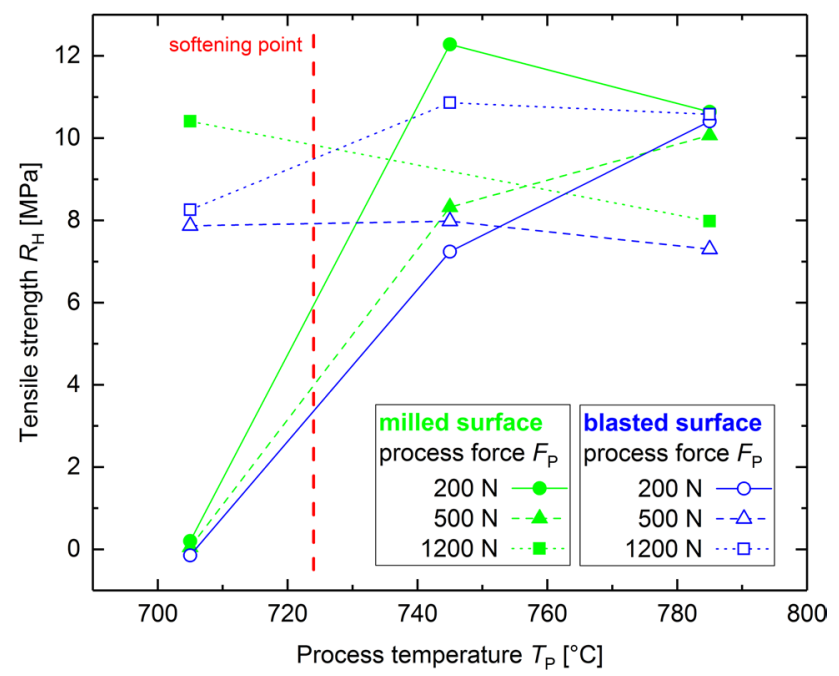

Fig. 4 Tensile strength as a function of process temperature for different process parameters

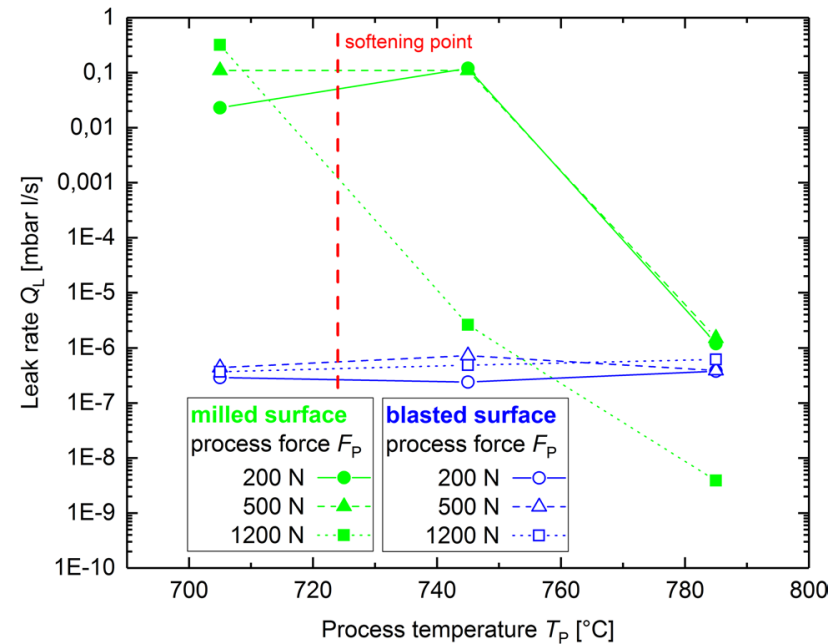

Fig. 5 Leak rate as a function of process temperature for different process parameters

of adhesion. Adhesion can roughly be subdivided into mechanical adhesion and specific adhesion [17]. Mechanical adhesion is based on interlocking, where the first material fills voids, pores, or undercuts of the second material.
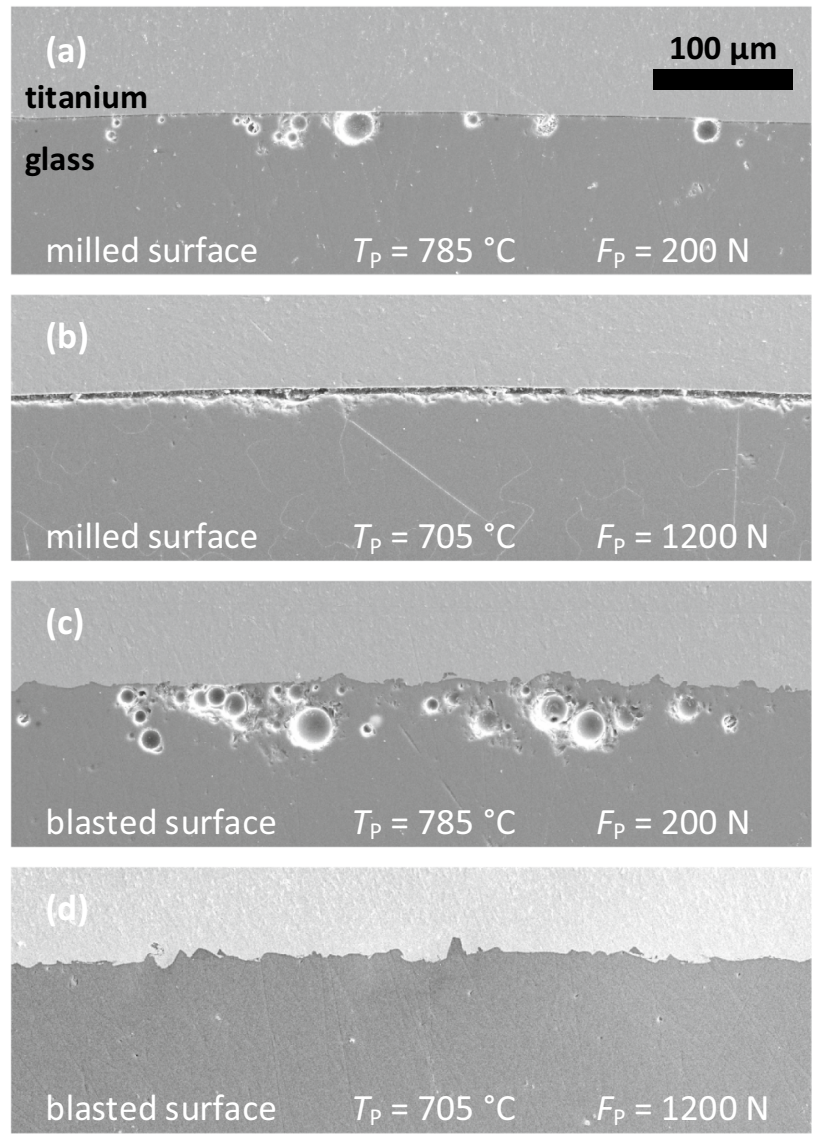

Fig. 6 Cross-sectional SEM image of glass-titanium bond interfaces 
Specific adhesion is based on electrostatic and molecular forces such as chemical forces or van der Waals forces. It describes adhesion between flat surfaces.

Oxide layers, such as $\mathrm{TiO}_{2}$, can prevent glass-to-metal bonding. The samples were cleaned as described by Donald [1] and bonded directly after machining and blasting to avoid the negative influence of titanium oxide layers. In the SEM images in Fig. $6 c$, d, the blasted titanium surface appears rough compared to the milled surface in Fig. 6a, b. However, undercuts can be excluded since tensile tests checked the adhesion using a test force perpendicular to the interface. Thus, mechanical adhesion can be neglected. However, specific adhesion does not require a roughened surface but can be increased by roughening the surface as it increases the surface area of the interface between the two materials.

In general, higher process temperatures, higher process forces, and a rough titanium surface lead to better results for both tensile strength and seal quality defined by leak rate. All samples with blasted surfaces are hermetic with leak rates lower than $1 \cdot 10^{-6} \mathrm{mbar} \cdot \mathrm{l} / \mathrm{s}$ and show high tensile strength values, with one exception $\left(T_{P}=705^{\circ} \mathrm{C}\right.$, $F_{p}=200 N$ ). The increased surface area can explain this effect due to the roughened metal surface.

In the SEM images in Fig. 6a, c, d, the materials are in close contact, and no visible gap can be detected. The observed seal quality is verified by the high tensile strength and low leak rate measured in samples produced with the same process parameters. In contrast, Fig. 6b shows a gap of approx. $5 \mu \mathrm{m}$ between glass and titanium. Accordingly, the measured leak rate is high. However, the parameter combination resulted in a relatively high tensile strength of more than $10 \mathrm{MPa}$. As two different sample designs were used, it is possible that in this case, the resulting glass-to-titanium interfaces significantly deviate in the two designs, even though they were produced with the same process parameters.

The SEM images in Fig. 6 show an accumulation of circular shapes in the glass material close to the interface. The shapes only appear at high process temperatures of $T_{\mathrm{P}}=785^{\circ} \mathrm{C}$ and appear to have no influence on the seal quality. Further analyses showed that the shapes are spherical cavities in the glass sample that were exposed during the preparation of the SEM samples. Since the cavities do not appear in all samples, they must originate from the bonding process. These cavities may be explained by gases, which are produced in chemical reactions during the bonding process at higher temperatures. However, no literature references were found indicating such effects. Another explanation for the cavities is the inclusion of argon gas, which fills the process chamber during the bonding process at low pressure. Edelmann [18] reported this effect for a similar hot embossing process.

\subsection{Conclusion}

The basic applicability of the novel bonding method has already been demonstrated by Winkler et al. [13]. However, other boundary conditions from relevant applications restrict the parameter field of the process. For example, optical functional surfaces such as lenses are typically molded below the softening point due to tool adhesion. Moreover, the design does not allow the application of high process forces in narrow electrical feedthroughs. Therefore, an optimum combination of process parameters has to be chosen depending on the application.

Results with low tensile strength and high leak rates occur at low process temperatures and low process forces. Due to the high viscosity of glass, more time is required for the glass to form a uniform interface to the metal. As all experiments were performed with the same process time of $15 \mathrm{~s}$, the glass could not cover the metal surface entirely at low temperatures and low process forces, which strongly reduced the seal quality. If it becomes necessary to realize the connection at temperatures below the softening point, the surface must be roughened, and high pressing loads have to be applied (see Figs. 4, 5). Longer process times are rarely possible due to poor demolding. Good mechanical adhesion was achieved at the high process force applied for the parameter combination in Fig. 6b. However, this good mechanical adhesion was not evenly distributed over the entire bond surface, so that no hermetic seal quality was achieved.

In all other applications, bonding should take place above the softening point. In this case, the clean and smooth surface shows no functional disadvantages regarding strength and hermetic seal quality if the process force is sufficient. However, too high process temperatures lead to bubble formation at the interface. Ideally, the process window's central area is derived at a bonding temperature just above the softening point and at pressing loads above $2.5 \mathrm{MPa}$, independent of the surface roughness.

\section{Summary and outlook}

An alternative process was tested and verified for bonding glass and titanium at medium temperatures by glass pressing. The most relevant process parameters were investigated, and the tensile strength and the leak rate were measured. Leak rates below $1 \cdot 10^{-6} \mathrm{mbar} \cdot \mathrm{l} / \mathrm{s}$ and tensile strengths of more than $7 \mathrm{MPa}$ were verified for most parameter combinations. The material interface was analyzed using SEM for selected parameter combinations.

Tensile strength and leak rate can be improved by higher process temperatures, and, to a smaller degree, by higher process forces. The blasted surface provides better 
bonding quality. Applying low process temperatures in combination with low process forces results in a mechanically weak interface that is not hermetic. In the SEM analysis, a large gap between the materials was observed for these samples. Using high temperatures led to cavities in the glass at the material interface, which did not negatively affect the seal quality.

It would be necessary to understand the adhesion mechanisms to obtain a deeper understanding of the process parameters' influence. While mechanical adhesion can be neglected, the type of forces affecting adhesion cannot be determined using the methods described in these investigations. Spectroscopic analysis such as auger electron spectroscopy [19] could be used to analyze the nature of the adhesion mechanism.

Acknowledgements The work was developed as part of the Fraunhofer pilot project Theranostic Implants-Approval-relevant development of key technologies for medicine to the authors thank the Fraunhofer-Gesellschaft for its support.

Funding Open Access funding enabled and organized by Projekt DEAL.

\section{Compliance with ethical standards}

Conflict of interest The authors declare that they have no conflict of interest.

Open Access This article is licensed under a Creative Commons Attribution 4.0 International License, which permits use, sharing, adaptation, distribution and reproduction in any medium or format, as long as you give appropriate credit to the original author(s) and the source, provide a link to the Creative Commons licence, and indicate if changes were made. The images or other third party material in this article are included in the article's Creative Commons licence, unless indicated otherwise in a credit line to the material. If material is not included in the article's Creative Commons licence and your intended use is not permitted by statutory regulation or exceeds the permitted use, you will need to obtain permission directly from the copyright holder. To view a copy of this licence, visit http://creativecommons .org/licenses/by/4.0/.

\section{References}

1. Donald I (2009) Glas-to-metal seals. Society of Glass Technology

2. Song $\mathrm{S}$ (2010) New glass-ceramic sealants for $\mathrm{Na} / \mathrm{S}$ battery. J Solid State Electrochem 14(9):S1735-S1740
3. Gomez-Vega J et al (2001) Bioactive glass-mesoporous silica coatings on $\mathrm{Ti}_{6} \mathrm{Al}_{4} \mathrm{~V}$ through enameling and triblock-copolymertemplated sol-gel processing. J Biomed Mater Res A 56:382-389

4. Winkler S, Edelmann J, Welsch C, Ruff R (2017) Different encapsulation strategies for implanted electronics. Curr Dir Biomed Eng 3(2):725-728

5. Donald I, Mallinson P, Metcalfe B, Gerrard L, Fernie J (2011) Recent developments in the preparation, characterization and applications of glass-and glass-ceramic-to-metal seals and coatings. J Mater Sci 46:1975-2000

6. Pazo A, Saiz E, Tomsia A (1998) Silicate glass coatings on Ti-based implants. Acta Mater 46:2551-2558

7. Goldstein Jl et al (1995) Solid-state reactions and phase relations in the Ti-Si-O system at $1373 \mathrm{~K}$. J Am Ceram Soc 78:313-322

8. Passerone AP, Valbusa G, Biagini E (1977) The titanium-molten glass system: interactions and wetting. J Mater Sci 12:2465-2474

9. Kuckert H, Born C, Wagner G, Eifler D (2001) Helium-tight sealing of glass with metal by ultrasonic welding. Adv Eng Mater 3:903-905

10. Briand D, Weber P, De Rooij NF (2004) Bonding properties of metals anodically bonded to glass. Sens Actuators A Phys 114:543-549

11. Khandan O, Stark D, Chang A, Rao MP (2014) Wafer-scale titanium anodic bonding for microfluidic applications. Sens Actuators B Chem 205:244-248

12. Fraunhofer-Gesellschaft zur Förderung der angewandten Forschung e.V. (2017) Patent: Housing Component, and Method for Manufacturing Same. 2017-05-11. WO 2017/194685 A1

13. Winkler S, Edelmann J, Günther D, Wieland S, Selbmann F, Baum M, Schubert A (2019) Sealing of glass with titanium by glass pressing at the softening point. SN Appl Sci 1(6):562

14. Lopez-Esteban S, Saiz E, Fujino S, Oku T, Suganuma K, Tomsia AP (2003) Bioactive glass coatings for orthopedic metallic implants. J Eur Ceram Soc 23:2921-2930

15. Goller G (2004) The effect of bond coat on mechanical properties of plasma sprayed bioglass-titanium coatings. Ceram Int 30:351-355

16. Kutzke K (1998) Dichtheitsprüfungen und Lecksuche mit dem Helium-Leckdetektor: ein Leitfaden zum praktischen Einsatz bei der Qualitätssicherung und Wartung. Expert Verlag, Renningen

17. Bischof C, Possart W (1983) Adhäsion: theoretische und experimentelle Grundlagen. Akademie-Verlag, Berlin

18. Edelmann J (2012) Mikrostrukturierung von Flachglas durch Heißprägen. Diss. Technische Universität Chemnitz

19. Takahashi K, Ishii H, Takahashi Y, Nishiguchi K (1992) Valence auger analysis of the annealing effect on atomic interaction at titanium-sapphire, titanium-silica and silver-silica interfaces. Thin Solid Films 221:98-103

Publisher's Note Springer Nature remains neutral with regard to jurisdictional claims in published maps and institutional affiliations. 